\title{
Synthesis and Properties of Compressed Dihydrogen Complexes of Iridium: Theoretical and Spectroscopic Investigations
}

\author{
Ricard Gelabert, Miquel Moreno, José M. Lluch* and Agustí Lledós*
}

Departament de Química, Universitat Autònoma de Barcelona, 08193 Bellaterra,

Barcelona, Spain.

Vincent Pons and D. Michael Heinekey*

Department of Chemistry, University of Washington, Seattle, WA 98195-1700, USA

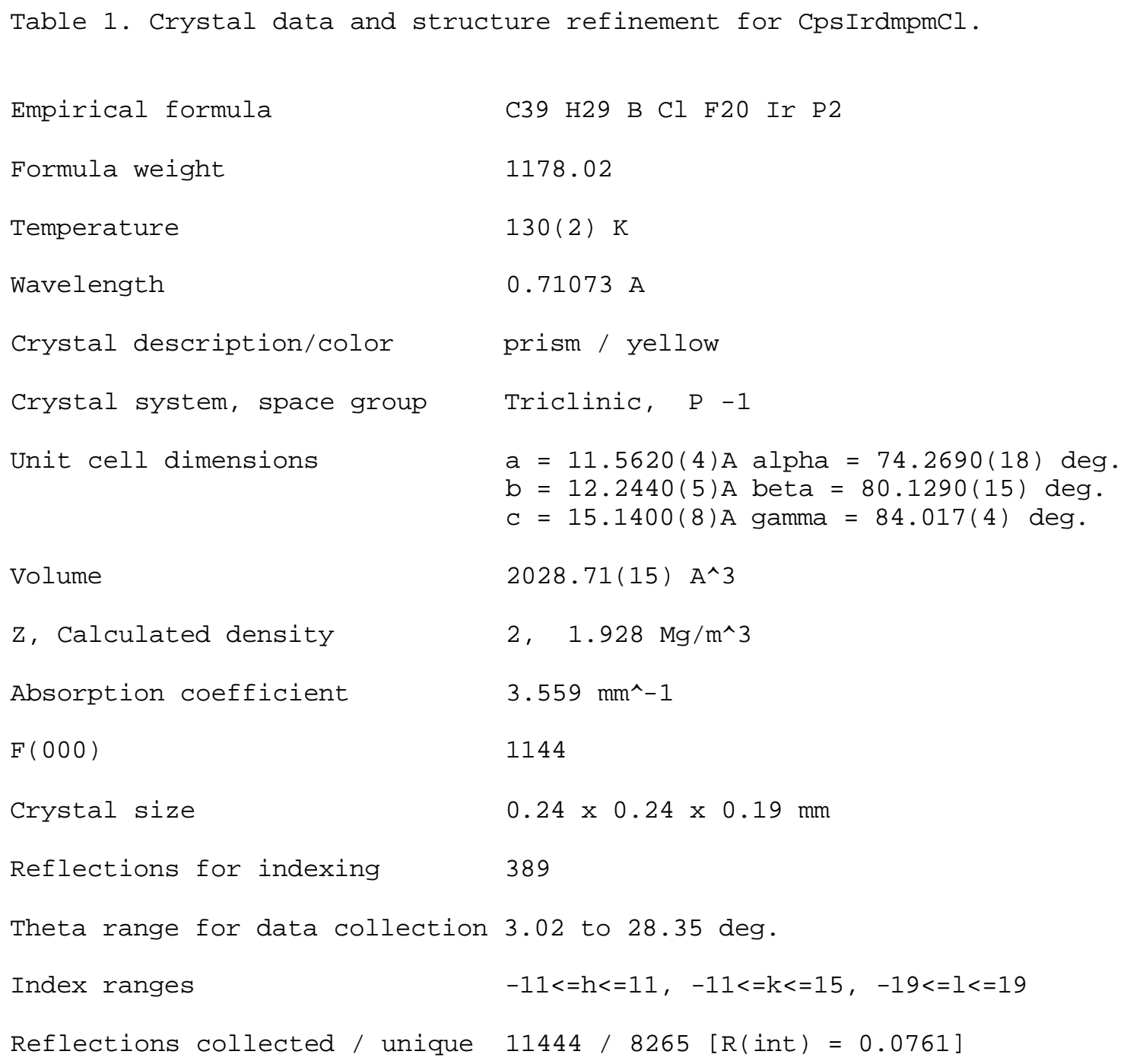




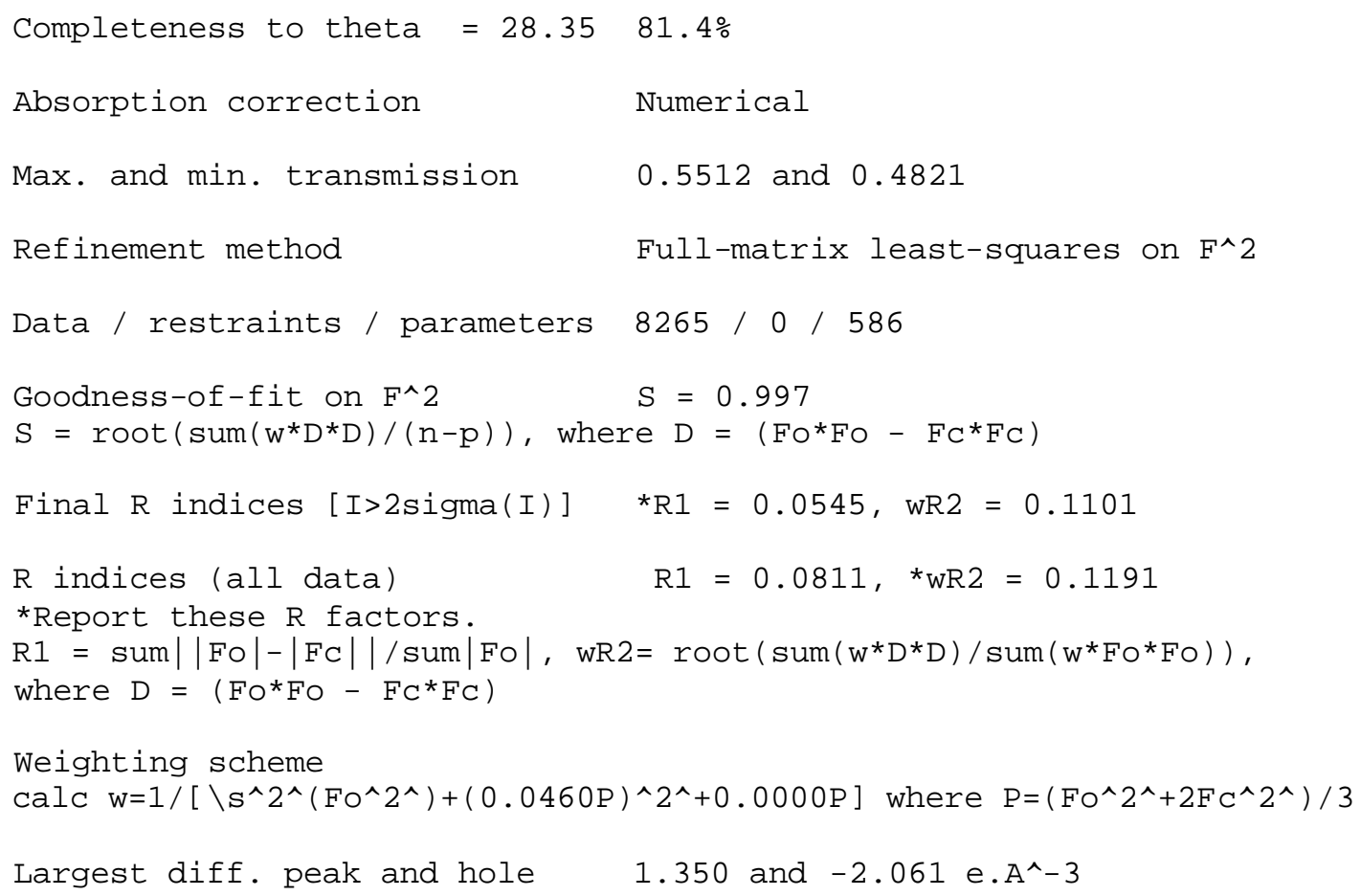


Table 2. Atomic coordinates ( $\left.\mathrm{x} 10^{\wedge} 4\right)$ and equivalent isotropic displacement parameters $\left(A^{\wedge} 2 \times 10^{\wedge} 3\right)$ for CpsIrdmpmCl.

$\mathrm{U}(\mathrm{eq})$ is defined as one third of the trace of the orthogonalized Uij tensor.

\begin{tabular}{|c|c|c|c|c|}
\hline & $\mathrm{x}$ & y & z & $\mathrm{U}(\mathrm{eq})$ \\
\hline $\operatorname{Ir}(1)$ & $1190(1)$ & $2126(1)$ & $2519(1)$ & $15(1)$ \\
\hline $\mathrm{Cl}(1)$ & $-380(1)$ & $868(2)$ & $3048(1)$ & $23(1)$ \\
\hline $\mathrm{P}(1)$ & $2270(1)$ & $664(2)$ & $3380(1)$ & $20(1)$ \\
\hline$P(2)$ & $1942(2)$ & $914(2)$ & $1617(1)$ & $20(1)$ \\
\hline$F(24)$ & $3183(4)$ & $-2750(4)$ & $7887(4)$ & $43(1)$ \\
\hline$F(56)$ & $1938(3)$ & $4227(4)$ & $5854(3)$ & $25(1)$ \\
\hline$F(36)$ & $4818(3)$ & $757(4)$ & $8434(3)$ & $23(1)$ \\
\hline$F(52)$ & $5322(3)$ & $1789(4)$ & $6503(3)$ & $25(1)$ \\
\hline$F(45)$ & $-910(3)$ & $3373(4)$ & $9127(3)$ & $33(1)$ \\
\hline$F(46)$ & $1382(3)$ & $2876(4)$ & $8985(2)$ & $23(1)$ \\
\hline$F(22)$ & $2325(3)$ & $501(4)$ & $9011(3)$ & $27(1)$ \\
\hline$F(26)$ & $3608(3)$ & $826(4)$ & $5811(3)$ & $24(1)$ \\
\hline$F(42)$ & $1307(3)$ & $2020(4)$ & $6124(2)$ & $23(1)$ \\
\hline$F(43)$ & $-989(3)$ & $2554(4)$ & $6272(3)$ & $30(1)$ \\
\hline$F(32)$ & $2784(3)$ & $4383(3)$ & $7670(3)$ & $24(1)$ \\
\hline$F(34)$ & $5804(4)$ & $3730(4)$ & $9490(3)$ & $35(1)$ \\
\hline$F(54)$ & $5358(4)$ & $4577(4)$ & $3692(3)$ & $42(1)$ \\
\hline$F(33)$ & $4096(3)$ & $5117(4)$ & $8648(3)$ & $27(1)$ \\
\hline$F(55)$ & $3094(4)$ & $5234(4)$ & $4233(3)$ & $35(1)$ \\
\hline$F(35)$ & $6136(3)$ & $1539(4)$ & $9347(3)$ & $33(1)$ \\
\hline$F(23)$ & $2394(4)$ & $-1742(4)$ & $9313(3)$ & $41(1)$ \\
\hline F (53) & $6479(3)$ & $2881(4)$ & $4879(3)$ & $34(1)$ \\
\hline$C(2)$ & $1016(6)$ & $3555(6)$ & $3194(4)$ & $19(2)$ \\
\hline$F(44)$ & $-2143(3)$ & $3234(4)$ & $7778(3)$ & $41(1)$ \\
\hline$C(3)$ & $-27(6)$ & $3611(6)$ & $2794(5)$ & $20(2)$ \\
\hline$C(41)$ & $1498(6)$ & $2490(6)$ & $7511(4)$ & $19(2)$ \\
\hline$C(52)$ & $4727(6)$ & $2642(7)$ & $5955(5)$ & $22(2)$ \\
\hline$C(24)$ & $3110(6)$ & $-1613(7)$ & $7729(6)$ & $32(2)$ \\
\hline$C(12)$ & $1672(6)$ & $-120(7)$ & $4517(5)$ & $29(2)$ \\
\hline C (33) & $4268(6)$ & $4047(6)$ & $8581(4)$ & $19(2)$ \\
\hline$C(46)$ & $854(6)$ & $2800(6)$ & $8261(5)$ & $21(2)$ \\
\hline$C(35)$ & $5289(6)$ & $2243(7)$ & $8934(4)$ & $22(2)$ \\
\hline$C(36)$ & $4597(5)$ & $1855(6)$ & $8446(4)$ & $18(2)$ \\
\hline$C(53)$ & $5339(6)$ & $3174(7)$ & $5117(5)$ & $27(2)$ \\
\hline$C(1)$ & $2012(5)$ & $3677(6)$ & $2435(4)$ & $19(2)$ \\
\hline$C(42)$ & $804(5)$ & $2367(6)$ & $6884(4)$ & $18(2)$ \\
\hline C ( 32$)$ & $3590(5)$ & $3639(6)$ & $8088(4)$ & $18(2)$ \\
\hline$C(8)$ & $-1231(5)$ & $3487(7)$ & $3334(5)$ & $24(2)$ \\
\hline$C(14)$ & $1001(6)$ & $365(7)$ & $1027(5)$ & $27(2)$ \\
\hline$C(26)$ & $3324(6)$ & $234(7)$ & $6701(5)$ & $23(2)$ \\
\hline C (31) & $3703(5)$ & $2528(6)$ & $8000(4)$ & $14(1)$ \\
\hline$C(21)$ & $3008(5)$ & $801(6)$ & $7405(5)$ & $18(2)$ \\
\hline$C(43)$ & $-397(6)$ & $2637(6)$ & $6949(5)$ & $22(2)$ \\
\hline$C(4)$ & $283(6)$ & $3734(6)$ & $1815(5)$ & $19(2)$ \\
\hline C (34) & $5138(6)$ & $3356(7)$ & $9006(5)$ & $22(2)$ \\
\hline$C(56)$ & 3051 (6) & $3811(6)$ & $5650(5)$ & $20(2)$ \\
\hline
\end{tabular}




\begin{tabular}{|c|c|c|c|c|}
\hline$C(13)$ & $2418(6)$ & $-237(7)$ & $2575(5)$ & $25(2)$ \\
\hline$C(25)$ & $3383(6)$ & $-930(7)$ & $6860(5)$ & $26(2)$ \\
\hline$C(22)$ & $2684(6)$ & $65(7)$ & $8266(5)$ & $21(2)$ \\
\hline$C(7)$ & $1086(6)$ & $3499(8)$ & $4178(5)$ & $32(2)$ \\
\hline$C(23)$ & $2725(6)$ & $-1098(7)$ & $8446(5)$ & $26(2)$ \\
\hline$C(10)$ & $2246(6)$ & $4118(7)$ & $646(5)$ & $24(2)$ \\
\hline$C(54)$ & $4775(7)$ & $4032(7)$ & $4516(5)$ & $27(2)$ \\
\hline$C(15)$ & $3242(6)$ & $1282(7)$ & $763(5)$ & $27(2)$ \\
\hline$C(51)$ & $3545(6)$ & $2892(6)$ & $6264(4)$ & $18(2)$ \\
\hline$C(45)$ & $-347(6)$ & $3057(7)$ & $8370(5)$ & $26(2)$ \\
\hline$C(44)$ & $-979(6)$ & $2980(6)$ & $7694(5)$ & $24(2)$ \\
\hline$C(5)$ & $1547(6)$ & $3822(6)$ & $1596(5)$ & $20(2)$ \\
\hline$C(6)$ & $3252(6)$ & $3859(7)$ & $2510(5)$ & $26(2)$ \\
\hline$C(55)$ & $3636(7)$ & $4373(6)$ & $4787(5)$ & $23(2)$ \\
\hline$C(11)$ & $3738(6)$ & $908(7)$ & 3491 (5) & $31(2)$ \\
\hline $\mathrm{B}(1)$ & $2928(6)$ & $2175(7)$ & 7301 (5) & $16(2)$ \\
\hline$C(9)$ & $-553(6)$ & $3907(7)$ & 1141 (5) & $30(2)$ \\
\hline$F(25)$ & $3729(3)$ & $-1403(4)$ & $6142(3)$ & $36(1)$ \\
\hline
\end{tabular}


Table 3. Bond lengths [A] and angles [deg] for CpsIrdmpmcl.

\begin{tabular}{|c|c|}
\hline $\operatorname{Ir}(1)-C(1)$ & $2.176(7)$ \\
\hline $\operatorname{Ir}(1)-C(5)$ & $2.202(7)$ \\
\hline $\operatorname{Ir}(1)-C(4)$ & $2.220(7)$ \\
\hline $\operatorname{Ir}(1)-C(2)$ & $2.231(7)$ \\
\hline $\operatorname{Ir}(1)-C(3)$ & $2.266(7)$ \\
\hline $\operatorname{Ir}(1)-P(2)$ & $2.2904(18)$ \\
\hline $\operatorname{Ir}(1)-P(1)$ & $2.2989(18)$ \\
\hline $\operatorname{Ir}(1)-\operatorname{Cl}(1)$ & $2.3917(16)$ \\
\hline$P(1)-C(12)$ & $1.788(7)$ \\
\hline$P(1)-C(11)$ & $1.797(7)$ \\
\hline$P(1)-C(13)$ & $1.831(8)$ \\
\hline$P(1)-P(2)$ & $2.691(2)$ \\
\hline$P(2)-C(14)$ & $1.802(7)$ \\
\hline$P(2)-C(15)$ & $1.815(7)$ \\
\hline$P(2)-C(13)$ & $1.843(7)$ \\
\hline$F(24)-C(24)$ & $1.343(9)$ \\
\hline$F(56)-C(56)$ & $1.350(8)$ \\
\hline$F(36)-C(36)$ & $1.346(8)$ \\
\hline$F(52)-C(52)$ & $1.352(8)$ \\
\hline$F(45)-C(45)$ & $1.348(8)$ \\
\hline$F(46)-C(46)$ & $1.370(7)$ \\
\hline$F(22)-C(22)$ & $1.359(8)$ \\
\hline$F(26)-C(26)$ & $1.348(8)$ \\
\hline$F(42)-C(42)$ & $1.352(7)$ \\
\hline$F(43)-C(43)$ & $1.357(8)$ \\
\hline$F(32)-C(32)$ & $1.346(7)$ \\
\hline$F(34)-C(34)$ & $1.337(7)$ \\
\hline$F(54)-C(54)$ & $1.347(8)$ \\
\hline$F(33)-C(33)$ & $1.332(8)$ \\
\hline$F(55)-C(55)$ & $1.331(8)$ \\
\hline$F(35)-C(35)$ & $1.354(8)$ \\
\hline$F(23)-C(23)$ & $1.350(8)$ \\
\hline$F(53)-C(53)$ & $1.343(8)$ \\
\hline$C(2)-C(3)$ & $1.427(9)$ \\
\hline$C(2)-C(1)$ & $1.470(9)$ \\
\hline$C(2)-C(7)$ & $1.489(9)$ \\
\hline$F(44)-C(44)$ & $1.342(7)$ \\
\hline$C(3)-C(4)$ & $1.434(9)$ \\
\hline$C(3)-C(8)$ & $1.488(9)$ \\
\hline$C(41)-C(46)$ & $1.369(9)$ \\
\hline$C(41)-C(42)$ & $1.390(9)$ \\
\hline $\begin{array}{l}C(41)-B(1) \\
C(52)-C(53)\end{array}$ & $\begin{array}{l}1.654(9) \\
1.369(10)\end{array}$ \\
\hline$C(52)-C(51)$ & $1.397(9)$ \\
\hline$C(24)-C(25)$ & $1.358(11)$ \\
\hline$C(24)-C(23)$ & $1.386(11)$ \\
\hline $\mathrm{C}(12)-\mathrm{H}(12 \mathrm{~A})$ & 0.9800 \\
\hline $\mathrm{C}(12)-\mathrm{H}(12 \mathrm{~B})$ & 0.9800 \\
\hline $\mathrm{C}(12)-\mathrm{H}(12 \mathrm{C})$ & 0.9800 \\
\hline$C(33)-C(34)$ & $1.375(10)$ \\
\hline$C(33)-C(32)$ & $1.381(9)$ \\
\hline$C(46)-C(45)$ & $1.384(10)$ \\
\hline$C(35)-C(36)$ & $1.373(9)$ \\
\hline
\end{tabular}




\begin{tabular}{|c|c|}
\hline$C(35)-C(34)$ & $1.387(10)$ \\
\hline$C(36)-C(31)$ & $1.401(9)$ \\
\hline$C(53)-C(54)$ & $1.378(11)$ \\
\hline$C(1)-C(5)$ & $1.424(9)$ \\
\hline$C(1)-C(6)$ & $1.501(9)$ \\
\hline$C(42)-C(43)$ & $1.386(10)$ \\
\hline$C(32)-C(31)$ & $1.394(9)$ \\
\hline $\mathrm{C}(8)-\mathrm{H}(8 \mathrm{~A})$ & 0.9800 \\
\hline $\mathrm{C}(8)-\mathrm{H}(8 \mathrm{~B})$ & 0.9800 \\
\hline $\mathrm{C}(8)-\mathrm{H}(8 \mathrm{C})$ & 0.9800 \\
\hline $\mathrm{C}(14)-\mathrm{H}(14 \mathrm{~A})$ & 0.9800 \\
\hline $\mathrm{C}(14)-\mathrm{H}(14 \mathrm{~B})$ & 0.9800 \\
\hline $\mathrm{C}(14)-\mathrm{H}(14 \mathrm{C})$ & 0.9800 \\
\hline$C(26)-C(25)$ & $1.376(11)$ \\
\hline$C(26)-C(21)$ & $1.402(9)$ \\
\hline$C(31)-B(1)$ & $1.662(9)$ \\
\hline$C(21)-C(22)$ & $1.385(10)$ \\
\hline $\mathrm{C}(21)-\mathrm{B}(1)$ & $1.641(11)$ \\
\hline$C(43)-C(44)$ & $1.356(10)$ \\
\hline$C(4)-C(5)$ & $1.450(9)$ \\
\hline$C(4)-C(9)$ & $1.484(9)$ \\
\hline$C(56)-C(51)$ & $1.392(10)$ \\
\hline$C(56)-C(55)$ & $1.395(10)$ \\
\hline $\mathrm{C}(13)-\mathrm{H}(13 \mathrm{~A})$ & 0.9900 \\
\hline $\mathrm{C}(13)-\mathrm{H}(13 \mathrm{~B})$ & 0.9900 \\
\hline$C(25)-F(25)$ & $1.350(8)$ \\
\hline$C(22)-C(23)$ & $1.372(11)$ \\
\hline $\mathrm{C}(7)-\mathrm{H}(7 \mathrm{~A})$ & 0.9800 \\
\hline$C(7)-H(7 B)$ & 0.9800 \\
\hline $\mathrm{C}(7)-\mathrm{H}(7 \mathrm{C})$ & 0.9800 \\
\hline$C(10)-C(5)$ & $1.494(9)$ \\
\hline $\mathrm{C}(10)-\mathrm{H}(10 \mathrm{~A})$ & 0.9800 \\
\hline $\mathrm{C}(10)-\mathrm{H}(10 \mathrm{~B})$ & 0.9800 \\
\hline $\mathrm{C}(10)-\mathrm{H}(10 \mathrm{C})$ & 0.9800 \\
\hline$C(54)-C(55)$ & $1.367(10)$ \\
\hline $\mathrm{C}(15)-\mathrm{H}(15 \mathrm{~A})$ & 0.9800 \\
\hline $\mathrm{C}(15)-\mathrm{H}(15 \mathrm{~B})$ & 0.9800 \\
\hline $\mathrm{C}(15)-\mathrm{H}(15 \mathrm{C})$ & 0.9800 \\
\hline$C(51)-B(1)$ & $1.657(10)$ \\
\hline$C(45)-C(44)$ & $1.383(10)$ \\
\hline$C(6)-H(6 A)$ & 0.9800 \\
\hline $\mathrm{C}(6)-\mathrm{H}(6 \mathrm{~B})$ & 0.9800 \\
\hline$C(6)-H(6 C)$ & 0.9800 \\
\hline $\mathrm{C}(11)-\mathrm{H}(11 \mathrm{~A})$ & 0.9800 \\
\hline $\mathrm{C}(11)-\mathrm{H}(11 \mathrm{~B})$ & 0.9800 \\
\hline $\mathrm{C}(11)-\mathrm{H}(11 \mathrm{C})$ & 0.9800 \\
\hline $\mathrm{C}(9)-\mathrm{H}(9 \mathrm{~A})$ & 0.9800 \\
\hline $\mathrm{C}(9)-\mathrm{H}(9 \mathrm{~B})$ & 0.9800 \\
\hline $\mathrm{C}(9)-\mathrm{H}(9 \mathrm{C})$ & 0.9800 \\
\hline$C(1)-\operatorname{Ir}(1)-C(5)$ & $38.0(2)$ \\
\hline$C(1)-\operatorname{Ir}(1)-C(4)$ & $64.2(3)$ \\
\hline$C(5)-\operatorname{Ir}(1)-C(4)$ & $38.3(2)$ \\
\hline$C(1)-\operatorname{Ir}(1)-C(2)$ & $38.9(2)$ \\
\hline$C(5)-\operatorname{Ir}(1)-C(2)$ & $63.5(2)$ \\
\hline$C(4)-\operatorname{Ir}(1)-C(2)$ & $63.2(2)$ \\
\hline$C(1)-\operatorname{Ir}(1)-C(3)$ & $63.3(2)$ \\
\hline
\end{tabular}




\begin{tabular}{|c|c|}
\hline$C(5)-\operatorname{Ir}(1)-C(3)$ & $62.5(2)$ \\
\hline$C(4)-\operatorname{Ir}(1)-C(3)$ & $37.3(2)$ \\
\hline$C(2)-\operatorname{Ir}(1)-C(3)$ & $37.0(2)$ \\
\hline $\mathrm{C}(1)-\operatorname{Ir}(1)-\mathrm{P}(2)$ & $122.60(18)$ \\
\hline$C(5)-\operatorname{Ir}(1)-P(2)$ & $103.76(18)$ \\
\hline $\mathrm{C}(4)-\operatorname{Ir}(1)-\mathrm{P}(2)$ & $116.25(18)$ \\
\hline $\mathrm{C}(2)-\operatorname{Ir}(1)-\mathrm{P}(2)$ & $161.26(18)$ \\
\hline $\mathrm{C}(3)-\operatorname{Ir}(1)-\mathrm{P}(2)$ & $151.37(17)$ \\
\hline $\mathrm{C}(1)-\operatorname{Ir}(1)-\mathrm{P}(1)$ & $106.15(18)$ \\
\hline$C(5)-\operatorname{Ir}(1)-P(1)$ & $135.74(18)$ \\
\hline $\mathrm{C}(4)-\operatorname{Ir}(1)-\mathrm{P}(1)$ & $169.54(19)$ \\
\hline $\mathrm{C}(2)-\operatorname{Ir}(1)-\mathrm{P}(1)$ & $106.90(18)$ \\
\hline $\mathrm{C}(3)-\operatorname{Ir}(1)-\mathrm{P}(1)$ & $136.10(17)$ \\
\hline$P(2)-\operatorname{Ir}(1)-P(1)$ & $71.81(6)$ \\
\hline$C(1)-\operatorname{Ir}(1)-C 1(1)$ & $152.82(18)$ \\
\hline$C(5)-\operatorname{Ir}(1)-C 1(1)$ & $139.42(18)$ \\
\hline$C(4)-\operatorname{Ir}(1)-C 1(1)$ & $102.26(18)$ \\
\hline$C(2)-\operatorname{Ir}(1)-C l(1)$ & $114.39(18)$ \\
\hline$C(3)-\operatorname{Ir}(1)-C 1(1)$ & $91.40(18)$ \\
\hline $\mathrm{P}(2)-\operatorname{Ir}(1)-\mathrm{Cl}(1)$ & $84.29(6)$ \\
\hline $\mathrm{P}(1)-\operatorname{Ir}(1)-\mathrm{Cl}(1)$ & $84.75(6)$ \\
\hline$C(12)-P(1)-C(11)$ & $104.2(3)$ \\
\hline$C(12)-P(1)-C(13)$ & $109.6(4)$ \\
\hline$C(11)-P(1)-C(13)$ & $106.4(4)$ \\
\hline $\mathrm{C}(12)-\mathrm{P}(1)-\operatorname{Ir}(1)$ & $121.4(2)$ \\
\hline $\mathrm{C}(11)-\mathrm{P}(1)-\operatorname{Ir}(1)$ & $118.4(3)$ \\
\hline$C(13)-P(1)-\operatorname{Ir}(1)$ & $95.5(2)$ \\
\hline $\mathrm{C}(12)-\mathrm{P}(1)-\mathrm{P}(2)$ & $137.9(3)$ \\
\hline $\mathrm{C}(11)-\mathrm{P}(1)-\mathrm{P}(2)$ & $113.7(3)$ \\
\hline $\mathrm{C}(13)-\mathrm{P}(1)-\mathrm{P}(2)$ & $43.1(2)$ \\
\hline $\operatorname{Ir}(1)-\mathrm{P}(1)-\mathrm{P}(2)$ & $53.95(5)$ \\
\hline $\mathrm{C}(14)-\mathrm{P}(2)-\mathrm{C}(15)$ & $104.2(3)$ \\
\hline$C(14)-P(2)-C(13)$ & $109.4(4)$ \\
\hline$C(15)-P(2)-C(13)$ & $106.6(3)$ \\
\hline $\mathrm{C}(14)-\mathrm{P}(2)-\operatorname{Ir}(1)$ & $120.7(3)$ \\
\hline$C(15)-P(2)-\operatorname{Ir}(1)$ & $119.1(3)$ \\
\hline$C(13)-P(2)-\operatorname{Ir}(1)$ & $95.5(2)$ \\
\hline $\mathrm{C}(14)-\mathrm{P}(2)-\mathrm{P}(1)$ & $137.1(2)$ \\
\hline $\mathrm{C}(15)-\mathrm{P}(2)-\mathrm{P}(1)$ & $114.3(3)$ \\
\hline $\mathrm{C}(13)-\mathrm{P}(2)-\mathrm{P}(1)$ & $42.7(2)$ \\
\hline $\operatorname{Ir}(1)-\mathrm{P}(2)-\mathrm{P}(1)$ & $54.24(5)$ \\
\hline$C(3)-C(2)-C(1)$ & $107.2(5)$ \\
\hline$C(3)-C(2)-C(7)$ & $126.9(6)$ \\
\hline$C(1)-C(2)-C(7)$ & $125.7(6)$ \\
\hline$C(3)-C(2)-\operatorname{Ir}(1)$ & $72.9(4)$ \\
\hline$C(1)-C(2)-\operatorname{Ir}(1)$ & $68.5(4)$ \\
\hline$C(7)-C(2)-\operatorname{Ir}(1)$ & $128.2(6)$ \\
\hline$C(2)-C(3)-C(4)$ & $109.3(6)$ \\
\hline$C(2)-C(3)-C(8)$ & $124.3(6)$ \\
\hline$C(4)-C(3)-C(8)$ & $126.3(6)$ \\
\hline$C(2)-C(3)-\operatorname{Ir}(1)$ & $70.1(4)$ \\
\hline$C(4)-C(3)-\operatorname{Ir}(1)$ & $69.6(4)$ \\
\hline$C(8)-C(3)-\operatorname{Ir}(1)$ & $123.7(5)$ \\
\hline$C(46)-C(41)-C(42)$ & $112.8(6)$ \\
\hline$C(46)-C(41)-B(1)$ & $129.2(6)$ \\
\hline$C(42)-C(41)-B(1)$ & $117.9(6)$ \\
\hline$F(52)-C(52)-C(53)$ & $116.3(6)$ \\
\hline
\end{tabular}




\begin{tabular}{|c|c|}
\hline$F(52)-C(52)-C(51)$ & $118.6(6)$ \\
\hline$C(53)-C(52)-C(51)$ & $125.1(7)$ \\
\hline$F(24)-C(24)-C(25)$ & $121.2(8)$ \\
\hline$F(24)-C(24)-C(23)$ & $121.0(8)$ \\
\hline$C(25)-C(24)-C(23)$ & $117.8(8)$ \\
\hline $\mathrm{P}(1)-\mathrm{C}(12)-\mathrm{H}(12 \mathrm{~A})$ & 109.5 \\
\hline $\mathrm{P}(1)-\mathrm{C}(12)-\mathrm{H}(12 \mathrm{~B})$ & 109.5 \\
\hline $\mathrm{H}(12 \mathrm{~A})-\mathrm{C}(12)-\mathrm{H}(12 \mathrm{~B})$ & 109.5 \\
\hline $\mathrm{P}(1)-\mathrm{C}(12)-\mathrm{H}(12 \mathrm{C})$ & 109.5 \\
\hline $\mathrm{H}(12 \mathrm{~A})-\mathrm{C}(12)-\mathrm{H}(12 \mathrm{C})$ & 109.5 \\
\hline $\mathrm{H}(12 \mathrm{~B})-\mathrm{C}(12)-\mathrm{H}(12 \mathrm{C})$ & 109.5 \\
\hline$F(33)-C(33)-C(34)$ & $119.0(6)$ \\
\hline$F(33)-C(33)-C(32)$ & $120.6(6)$ \\
\hline$C(34)-C(33)-C(32)$ & $120.4(7)$ \\
\hline$C(41)-C(46)-F(46)$ & $120.9(6)$ \\
\hline$C(41)-C(46)-C(45)$ & $124.9(6)$ \\
\hline$F(46)-C(46)-C(45)$ & $114.2(6)$ \\
\hline$F(35)-C(35)-C(36)$ & $120.0(7)$ \\
\hline$F(35)-C(35)-C(34)$ & $119.2(6)$ \\
\hline$C(36)-C(35)-C(34)$ & $120.7(6)$ \\
\hline$F(36)-C(36)-C(35)$ & $115.3(6)$ \\
\hline $\mathrm{F}(36)-\mathrm{C}(36)-\mathrm{C}(31)$ & $121.2(6)$ \\
\hline$C(35)-C(36)-C(31)$ & $123.5(7)$ \\
\hline$F(53)-C(53)-C(52)$ & $121.0(8)$ \\
\hline$F(53)-C(53)-C(54)$ & $119.8(7)$ \\
\hline$C(52)-C(53)-C(54)$ & $119.2(7)$ \\
\hline$C(5)-C(1)-C(2)$ & $107.5(6)$ \\
\hline$C(5)-C(1)-C(6)$ & $126.0(6)$ \\
\hline$C(2)-C(1)-C(6)$ & $125.4(6)$ \\
\hline$C(5)-C(1)-\operatorname{Ir}(1)$ & $72.0(4)$ \\
\hline$C(2)-C(1)-\operatorname{Ir}(1)$ & $72.5(4)$ \\
\hline$C(6)-C(1)-\operatorname{Ir}(1)$ & $130.4(5)$ \\
\hline$F(42)-C(42)-C(43)$ & $115.6(6)$ \\
\hline$F(42)-C(42)-C(41)$ & $119.9(6)$ \\
\hline$C(43)-C(42)-C(41)$ & $124.4(6)$ \\
\hline$F(32)-C(32)-C(33)$ & $116.5(6)$ \\
\hline$F(32)-C(32)-C(31)$ & $119.5(6)$ \\
\hline$C(33)-C(32)-C(31)$ & $124.0(6)$ \\
\hline $\mathrm{C}(3)-\mathrm{C}(8)-\mathrm{H}(8 \mathrm{~A})$ & 109.5 \\
\hline$C(3)-C(8)-H(8 B)$ & 109.5 \\
\hline $\mathrm{H}(8 \mathrm{~A})-\mathrm{C}(8)-\mathrm{H}(8 \mathrm{~B})$ & 109.5 \\
\hline $\mathrm{C}(3)-\mathrm{C}(8)-\mathrm{H}(8 \mathrm{C})$ & 109.5 \\
\hline $\mathrm{H}(8 \mathrm{~A})-\mathrm{C}(8)-\mathrm{H}(8 \mathrm{C})$ & 109.5 \\
\hline $\mathrm{H}(8 \mathrm{~B})-\mathrm{C}(8)-\mathrm{H}(8 \mathrm{C})$ & 109.5 \\
\hline $\mathrm{P}(2)-\mathrm{C}(14)-\mathrm{H}(14 \mathrm{~A})$ & 109.5 \\
\hline $\mathrm{P}(2)-\mathrm{C}(14)-\mathrm{H}(14 \mathrm{~B})$ & 109.5 \\
\hline $\mathrm{H}(14 \mathrm{~A})-\mathrm{C}(14)-\mathrm{H}(14 \mathrm{~B})$ & 109.5 \\
\hline $\mathrm{P}(2)-\mathrm{C}(14)-\mathrm{H}(14 \mathrm{C})$ & 109.5 \\
\hline $\mathrm{H}(14 \mathrm{~A})-\mathrm{C}(14)-\mathrm{H}(14 \mathrm{C})$ & 109.5 \\
\hline $\mathrm{H}(14 \mathrm{~B})-\mathrm{C}(14)-\mathrm{H}(14 \mathrm{C})$ & 109.5 \\
\hline$F(26)-C(26)-C(25)$ & $116.0(6)$ \\
\hline$F(26)-C(26)-C(21)$ & $120.4(7)$ \\
\hline$C(25)-C(26)-C(21)$ & $123.6(7)$ \\
\hline$C(32)-C(31)-C(36)$ & $113.5(6)$ \\
\hline$C(32)-C(31)-B(1)$ & $119.4(6)$ \\
\hline$C(36)-C(31)-B(1)$ & $126.6(6)$ \\
\hline
\end{tabular}




\begin{tabular}{|c|c|}
\hline$C(22)-C(21)-C(26)$ & $112.5(7)$ \\
\hline$C(22)-C(21)-B(1)$ & $119.5(6)$ \\
\hline$C(26)-C(21)-B(1)$ & $127.9(6)$ \\
\hline$C(44)-C(43)-F(43)$ & $120.1(6)$ \\
\hline$C(44)-C(43)-C(42)$ & $119.7(6)$ \\
\hline$F(43)-C(43)-C(42)$ & $120.2(6)$ \\
\hline$C(3)-C(4)-C(5)$ & $107.1(6)$ \\
\hline$C(3)-C(4)-C(9)$ & $125.9(6)$ \\
\hline$C(5)-C(4)-C(9)$ & $126.5(6)$ \\
\hline$C(3)-C(4)-\operatorname{Ir}(1)$ & $73.1(4)$ \\
\hline$C(5)-C(4)-\operatorname{Ir}(1)$ & $70.2(4)$ \\
\hline$C(9)-C(4)-\operatorname{Ir}(1)$ & $128.6(5)$ \\
\hline$F(34)-C(34)-C(33)$ & $121.5(7)$ \\
\hline$F(34)-C(34)-C(35)$ & $120.7(6)$ \\
\hline$C(33)-C(34)-C(35)$ & $117.8(6)$ \\
\hline$F(56)-C(56)-C(51)$ & $121.5(6)$ \\
\hline$F(56)-C(56)-C(55)$ & $114.4(6)$ \\
\hline$C(51)-C(56)-C(55)$ & $124.1(7)$ \\
\hline$P(1)-C(13)-P(2)$ & $94.2(4)$ \\
\hline$P(1)-C(13)-H(13 A)$ & 112.9 \\
\hline$P(2)-C(13)-H(13 A)$ & 112.9 \\
\hline$P(1)-C(13)-H(13 B)$ & 112.9 \\
\hline $\mathrm{P}(2)-\mathrm{C}(13)-\mathrm{H}(13 \mathrm{~B})$ & 112.9 \\
\hline $\mathrm{H}(13 \mathrm{~A})-\mathrm{C}(13)-\mathrm{H}(13 \mathrm{~B})$ & 110.3 \\
\hline$F(25)-C(25)-C(24)$ & $119.4(7)$ \\
\hline$F(25)-C(25)-C(26)$ & $119.4(7)$ \\
\hline$C(24)-C(25)-C(26)$ & $121.2(7)$ \\
\hline$F(22)-C(22)-C(23)$ & $115.9(6)$ \\
\hline$F(22)-C(22)-C(21)$ & $118.9(7)$ \\
\hline$C(23)-C(22)-C(21)$ & $125.1(7)$ \\
\hline $\mathrm{C}(2)-\mathrm{C}(7)-\mathrm{H}(7 \mathrm{~A})$ & 109.5 \\
\hline $\mathrm{C}(2)-\mathrm{C}(7)-\mathrm{H}(7 \mathrm{~B})$ & 109.5 \\
\hline $\mathrm{H}(7 \mathrm{~A})-\mathrm{C}(7)-\mathrm{H}(7 \mathrm{~B})$ & 109.5 \\
\hline $\mathrm{C}(2)-\mathrm{C}(7)-\mathrm{H}(7 \mathrm{C})$ & 109.5 \\
\hline $\mathrm{H}(7 \mathrm{~A})-\mathrm{C}(7)-\mathrm{H}(7 \mathrm{C})$ & 109.5 \\
\hline $\mathrm{H}(7 \mathrm{~B})-\mathrm{C}(7)-\mathrm{H}(7 \mathrm{C})$ & 109.5 \\
\hline$F(23)-C(23)-C(22)$ & $120.6(7)$ \\
\hline$F(23)-C(23)-C(24)$ & $119.8(7)$ \\
\hline$C(22)-C(23)-C(24)$ & $119.6(7)$ \\
\hline $\mathrm{C}(5)-\mathrm{C}(10)-\mathrm{H}(10 \mathrm{~A})$ & 109.5 \\
\hline $\mathrm{C}(5)-\mathrm{C}(10)-\mathrm{H}(10 \mathrm{~B})$ & 109.5 \\
\hline $\mathrm{H}(10 \mathrm{~A})-\mathrm{C}(10)-\mathrm{H}(10 \mathrm{~B})$ & 109.5 \\
\hline$C(5)-C(10)-H(10 C)$ & 109.5 \\
\hline $\mathrm{H}(10 \mathrm{~A})-\mathrm{C}(10)-\mathrm{H}(10 \mathrm{C})$ & 109.5 \\
\hline $\mathrm{H}(10 \mathrm{~B})-\mathrm{C}(10)-\mathrm{H}(10 \mathrm{C})$ & 109.5 \\
\hline$F(54)-C(54)-C(55)$ & $120.0(7)$ \\
\hline$F(54)-C(54)-C(53)$ & $120.4(7)$ \\
\hline$C(55)-C(54)-C(53)$ & $119.4(6)$ \\
\hline$P(2)-C(15)-H(15 A)$ & 109.5 \\
\hline $\mathrm{P}(2)-\mathrm{C}(15)-\mathrm{H}(15 \mathrm{~B})$ & 109.5 \\
\hline $\mathrm{H}(15 \mathrm{~A})-\mathrm{C}(15)-\mathrm{H}(15 \mathrm{~B})$ & 109.5 \\
\hline$P(2)-C(15)-H(15 C)$ & 109.5 \\
\hline $\mathrm{H}(15 \mathrm{~A})-\mathrm{C}(15)-\mathrm{H}(15 \mathrm{C})$ & 109.5 \\
\hline $\mathrm{H}(15 \mathrm{~B})-\mathrm{C}(15)-\mathrm{H}(15 \mathrm{C})$ & 109.5 \\
\hline$C(56)-C(51)-C(52)$ & $112.7(6)$ \\
\hline$C(56)-C(51)-B(1)$ & $127.7(6)$ \\
\hline
\end{tabular}




\begin{tabular}{|c|c|}
\hline$C(52)-C(51)-B(1)$ & $119.4(6)$ \\
\hline$F(45)-C(45)-C(44)$ & $119.7(6)$ \\
\hline$F(45)-C(45)-C(46)$ & $121.0(6)$ \\
\hline$C(44)-C(45)-C(46)$ & $119.3(6)$ \\
\hline$F(44)-C(44)-C(43)$ & $120.9(7)$ \\
\hline$F(44)-C(44)-C(45)$ & $120.5(6)$ \\
\hline$C(43)-C(44)-C(45)$ & $118.6(6)$ \\
\hline$C(1)-C(5)-C(4)$ & $108.8(6)$ \\
\hline$C(1)-C(5)-C(10)$ & $124.7(6)$ \\
\hline$C(4)-C(5)-C(10)$ & $126.3(6)$ \\
\hline$C(1)-C(5)-\operatorname{Ir}(1)$ & $70.0(4)$ \\
\hline$C(4)-C(5)-\operatorname{Ir}(1)$ & $71.5(4)$ \\
\hline$C(10)-C(5)-\operatorname{Ir}(1)$ & $128.5(5)$ \\
\hline$C(1)-C(6)-H(6 A)$ & 109.5 \\
\hline $\mathrm{C}(1)-\mathrm{C}(6)-\mathrm{H}(6 \mathrm{~B})$ & 109.5 \\
\hline$H(6 A)-C(6)-H(6 B)$ & 109.5 \\
\hline$C(1)-C(6)-H(6 C)$ & 109.5 \\
\hline $\mathrm{H}(6 \mathrm{~A})-\mathrm{C}(6)-\mathrm{H}(6 \mathrm{C})$ & 109.5 \\
\hline $\mathrm{H}(6 \mathrm{~B})-\mathrm{C}(6)-\mathrm{H}(6 \mathrm{C})$ & 109.5 \\
\hline$F(55)-C(55)-C(54)$ & $120.3(7)$ \\
\hline$F(55)-C(55)-C(56)$ & $120.4(7)$ \\
\hline$C(54)-C(55)-C(56)$ & $119.3(7)$ \\
\hline $\mathrm{P}(1)-\mathrm{C}(11)-\mathrm{H}(11 \mathrm{~A})$ & 109.5 \\
\hline $\mathrm{P}(1)-\mathrm{C}(11)-\mathrm{H}(11 \mathrm{~B})$ & 109.5 \\
\hline $\mathrm{H}(11 \mathrm{~A})-\mathrm{C}(11)-\mathrm{H}(11 \mathrm{~B})$ & 109.5 \\
\hline $\mathrm{P}(1)-\mathrm{C}(11)-\mathrm{H}(11 \mathrm{C})$ & 109.5 \\
\hline $\mathrm{H}(11 \mathrm{~A})-\mathrm{C}(11)-\mathrm{H}(11 \mathrm{C})$ & 109.5 \\
\hline $\mathrm{H}(11 \mathrm{~B})-\mathrm{C}(11)-\mathrm{H}(11 \mathrm{C})$ & 109.5 \\
\hline $\mathrm{C}(21)-\mathrm{B}(1)-\mathrm{C}(41)$ & $103.3(5)$ \\
\hline$C(21)-B(1)-C(51)$ & $112.2(5)$ \\
\hline$C(41)-B(1)-C(51)$ & $112.9(6)$ \\
\hline$C(21)-B(1)-C(31)$ & $112.7(5)$ \\
\hline$C(41)-B(1)-C(31)$ & $114.1(5)$ \\
\hline$C(51)-B(1)-C(31)$ & $102.0(5)$ \\
\hline $\mathrm{C}(4)-\mathrm{C}(9)-\mathrm{H}(9 \mathrm{~A})$ & 109.5 \\
\hline $\mathrm{C}(4)-\mathrm{C}(9)-\mathrm{H}(9 \mathrm{~B})$ & 109.5 \\
\hline $\mathrm{H}(9 \mathrm{~A})-\mathrm{C}(9)-\mathrm{H}(9 \mathrm{~B})$ & 109.5 \\
\hline $\mathrm{C}(4)-\mathrm{C}(9)-\mathrm{H}(9 \mathrm{C})$ & 109.5 \\
\hline $\mathrm{H}(9 \mathrm{~A})-\mathrm{C}(9)-\mathrm{H}(9 \mathrm{C})$ & 109.5 \\
\hline $\mathrm{H}(9 \mathrm{~B})-\mathrm{C}(9)-\mathrm{H}(9 \mathrm{C})$ & 109.5 \\
\hline
\end{tabular}

Symmetry transformations used to generate equivalent atoms: 
Table 4. Anisotropic displacement parameters ( $A^{\wedge} 2 \times 10^{\wedge} 3$ ) for CpsIrdmpmCl. The anisotropic displacement factor exponent takes the form:

$-2 \mathrm{pi} 2\left[\mathrm{~h}{ }^{\wedge} 2 \mathrm{a}^{\star \wedge} 2 \mathrm{U} 11+\ldots+2 \mathrm{~h} k \mathrm{a}^{\star} \mathrm{b}\right.$ * U12 ]

\begin{tabular}{|c|c|c|c|c|c|c|}
\hline & U11 & U22 & U33 & U23 & U13 & U12 \\
\hline $\operatorname{Ir}(1)$ & $12(1)$ & $18(1)$ & $15(1)$ & $-2(1)$ & $-2(1)$ & $-1(1)$ \\
\hline $\mathrm{Cl}(1)$ & $14(1)$ & $26(1)$ & $26(1)$ & $-1(1)$ & $-2(1)$ & $-7(1)$ \\
\hline $\mathrm{P}(1)$ & $15(1)$ & $21(1)$ & $19(1)$ & $1(1)$ & $-3(1)$ & $2(1)$ \\
\hline$P(2)$ & $24(1)$ & $17(1)$ & $17(1)$ & $-2(1)$ & $-1(1)$ & $1(1)$ \\
\hline$F(24)$ & $44(3)$ & $17(3)$ & $68(3)$ & $-2(2)$ & $-23(2)$ & $-1(2)$ \\
\hline$F(56)$ & $28(2)$ & $23(3)$ & $24(2)$ & $-3(2)$ & $-8(2)$ & $-3(2)$ \\
\hline$F(36)$ & $24(2)$ & $18(2)$ & $29(2)$ & $-8(2)$ & $-11(2)$ & $3(2)$ \\
\hline$F(52)$ & $20(2)$ & $26(3)$ & $29(2)$ & $-8(2)$ & $-4(2)$ & $4(2)$ \\
\hline$F(45)$ & $23(2)$ & $43(3)$ & $30(2)$ & $-15(2)$ & $7(2)$ & $4(2)$ \\
\hline$F(46)$ & $24(2)$ & $30(3)$ & $16(2)$ & $-9(2)$ & $0(2)$ & $-3(2)$ \\
\hline$F(22)$ & $33(2)$ & $28(3)$ & $18(2)$ & $1(2)$ & $-4(2)$ & $-9(2)$ \\
\hline$F(26)$ & $30(2)$ & $22(3)$ & $22(2)$ & $-9(2)$ & $3(2)$ & $-7(2)$ \\
\hline$F(42)$ & $22(2)$ & $28(3)$ & $19(2)$ & $-7(2)$ & $-1(2)$ & $-2(2)$ \\
\hline$F(43)$ & $20(2)$ & $38(3)$ & $32(2)$ & $-4(2)$ & $-13(2)$ & $-3(2)$ \\
\hline$F(32)$ & $23(2)$ & $17(2)$ & $29(2)$ & $-2(2)$ & $-7(2)$ & $3(2)$ \\
\hline$F(34)$ & $33(3)$ & $37(3)$ & $41(3)$ & $-13(2)$ & $-17(2)$ & $-11(2)$ \\
\hline F (54) & $54(3)$ & $51(4)$ & $20(2)$ & $-8(2)$ & $10(2)$ & $-33(3)$ \\
\hline$F(33)$ & $34(2)$ & $15(3)$ & $36(2)$ & $-9(2)$ & $-8(2)$ & $-5(2)$ \\
\hline$F(55)$ & $56(3)$ & $22(3)$ & $27(2)$ & $5(2)$ & $-15(2)$ & $-17(2)$ \\
\hline$F(35)$ & $30(2)$ & $28(3)$ & $42(3)$ & $-6(2)$ & $-22(2)$ & $6(2)$ \\
\hline$F(23)$ & $53(3)$ & $30(3)$ & $40(3)$ & $8(2)$ & $-20(2)$ & $-17(2)$ \\
\hline F (53) & $25(2)$ & 41 (3) & $38(3)$ & $-23(2)$ & $14(2)$ & $-16(2)$ \\
\hline$C(2)$ & $23(4)$ & $20(4)$ & $16(3)$ & $-5(3)$ & $-8(3)$ & $1(3)$ \\
\hline$F(44)$ & $13(2)$ & $55(4)$ & $51(3)$ & $-9(3)$ & $-3(2)$ & $1(2)$ \\
\hline$C(3)$ & $30(4)$ & $7(4)$ & $21(4)$ & $4(3)$ & $-7(3)$ & $5(3)$ \\
\hline$C(41)$ & $17(4)$ & $13(4)$ & $21(4)$ & $2(3)$ & $0(3)$ & $-2(3)$ \\
\hline$C(52)$ & $19(4)$ & $28(5)$ & $22(4)$ & $-9(3)$ & $2(3)$ & $-11(3)$ \\
\hline$C(24)$ & $21(4)$ & $21(5)$ & $62(6)$ & $-9(4)$ & $-26(4)$ & $-6(3)$ \\
\hline$C(12)$ & $18(4)$ & $36(5)$ & $27(4)$ & $1(4)$ & $-5(3)$ & $8(3)$ \\
\hline C (33) & $23(4)$ & $10(4)$ & $22(4)$ & $-4(3)$ & $0(3)$ & $-2(3)$ \\
\hline$C(46)$ & $26(4)$ & $18(4)$ & $18(4)$ & $-4(3)$ & $-3(3)$ & $-6(3)$ \\
\hline$C(35)$ & $17(4)$ & $31(5)$ & $13(3)$ & $1(3)$ & $-4(2)$ & $-3(3)$ \\
\hline$C(36)$ & $16(4)$ & $17(4)$ & $21(4)$ & $-7(3)$ & $4(3)$ & $-5(3)$ \\
\hline C (53) & $22(4)$ & $35(5)$ & $31(4)$ & $-19(4)$ & $4(3)$ & $-14(3)$ \\
\hline$C(1)$ & $15(4)$ & $19(4)$ & $20(4)$ & $-1(3)$ & $-1(2)$ & $-5(3)$ \\
\hline$C(42)$ & $19(4)$ & $13(4)$ & $21(4)$ & $-1(3)$ & $-5(3)$ & $2(3)$ \\
\hline$C(32)$ & $11(3)$ & $25(4)$ & $14(3)$ & $-4(3)$ & $-1(2)$ & $1(3)$ \\
\hline$C(8)$ & $10(3)$ & $37(5)$ & $27(4)$ & $-14(4)$ & $4(3)$ & $3(3)$ \\
\hline C (14) & $37(4)$ & $23(5)$ & $19(4)$ & $0(3)$ & $-6(3)$ & $-2(3)$ \\
\hline$C(26)$ & $14(4)$ & $32(5)$ & $26(4)$ & $-11(3)$ & $-5(3)$ & $-4(3)$ \\
\hline C (31) & $14(3)$ & $11(4)$ & $17(3)$ & $-4(3)$ & $-2(2)$ & $-1(3)$ \\
\hline$C(21)$ & $13(3)$ & $19(4)$ & $24(4)$ & $-10(3)$ & $-3(2)$ & $0(3)$ \\
\hline$C(43)$ & $24(4)$ & $16(4)$ & $24(4)$ & $2(3)$ & $-6(3)$ & $-7(3)$ \\
\hline$C(4)$ & $20(4)$ & $12(4)$ & $21(4)$ & $2(3)$ & $-6(3)$ & $6(3)$ \\
\hline$C(34)$ & $18(4)$ & $27(5)$ & $23(4)$ & $-8(3)$ & $-4(3)$ & $-12(3)$ \\
\hline$C(56)$ & $20(4)$ & $22(4)$ & $20(4)$ & $-9(3)$ & $-1(3)$ & $-9(3)$ \\
\hline$C(13)$ & $31(4)$ & $20(5)$ & $19(4)$ & $3(3)$ & $0(3)$ & $-4(3)$ \\
\hline
\end{tabular}




\begin{tabular}{|c|c|c|c|c|c|c|}
\hline$C(25)$ & $20(4)$ & $24(5)$ & $44(5)$ & $-20(4)$ & $-10(3)$ & $-4(3)$ \\
\hline$C(22)$ & $15(4)$ & $27(5)$ & $19(4)$ & $-5(3)$ & $-1(2)$ & $-3(3)$ \\
\hline$C(7)$ & $31(4)$ & $52(6)$ & $18(4)$ & $-18(4)$ & $-4(3)$ & $-4(4)$ \\
\hline$C(23)$ & $31(4)$ & $20(5)$ & $26(4)$ & $4(3)$ & $-14(3)$ & $-9(3)$ \\
\hline$C(10)$ & $27(4)$ & $18(4)$ & $23(4)$ & $-2(3)$ & $5(3)$ & $-4(3)$ \\
\hline$C(54)$ & $41(5)$ & $27(5)$ & $17(4)$ & $-11(3)$ & $7(3)$ & $-23(4)$ \\
\hline$C(15)$ & $31(4)$ & $21(5)$ & $24(4)$ & $-8(3)$ & $8(3)$ & $5(3)$ \\
\hline C (51) & $24(4)$ & $18(4)$ & $15(3)$ & $-5(3)$ & $-7(3)$ & $-6(3)$ \\
\hline$C(45)$ & $12(4)$ & $32(5)$ & $29(4)$ & $-5(3)$ & $2(3)$ & $2(3)$ \\
\hline$C(44)$ & $10(4)$ & $20(5)$ & $34(4)$ & $2(3)$ & $0(3)$ & $4(3)$ \\
\hline$C(5)$ & $29(4)$ & $4(4)$ & $25(4)$ & $2(3)$ & $-4(3)$ & $-4(3)$ \\
\hline$C(6)$ & $27(4)$ & $22(5)$ & $32(4)$ & $-5(3)$ & $-11(3)$ & $-6(3)$ \\
\hline C (55) & $43(5)$ & $6(4)$ & $22(4)$ & $-1(3)$ & $-16(3)$ & $-4(3)$ \\
\hline$C(11)$ & $17(4)$ & $35(5)$ & $35(4)$ & $5(4)$ & $-13(3)$ & $0(3)$ \\
\hline B (1) & $20(4)$ & $15(5)$ & $13(4)$ & $-2(3)$ & $-3(3)$ & $-4(3)$ \\
\hline$C(9)$ & $21(4)$ & $39(6)$ & $30(4)$ & $-5(4)$ & $-15(3)$ & $3(3)$ \\
\hline$F(25)$ & $28(2)$ & $29(3)$ & $59(3)$ & $-29(3)$ & $-7(2)$ & $3(2)$ \\
\hline
\end{tabular}


Table 5. Hydrogen coordinates $\left(x 0^{\wedge} 4\right)$ and isotropic displacement parameters $\left(A^{\wedge} 2 \times 10^{\wedge} 3\right)$ for CpsIrdmpmCl.

\begin{tabular}{|c|c|c|c|c|}
\hline & $x$ & $y$ & $\mathrm{z}$ & $\mathrm{U}(\mathrm{eq})$ \\
\hline $\mathrm{H}(12 \mathrm{~A})$ & 951 & -455 & 4486 & 44 \\
\hline $\mathrm{H}(12 \mathrm{~B})$ & 1491 & 391 & 4927 & 44 \\
\hline $\mathrm{H}(12 \mathrm{C})$ & 2247 & -726 & 4760 & 44 \\
\hline $\mathrm{H}(8 \mathrm{~A})$ & -1193 & 2944 & 3938 & 37 \\
\hline $\mathrm{H}(8 \mathrm{~B})$ & -1735 & 3211 & 2992 & 37 \\
\hline $\mathrm{H}(8 \mathrm{C})$ & -1558 & 4227 & 3425 & 37 \\
\hline $\mathrm{H}(14 \mathrm{~A})$ & 754 & 974 & 513 & 41 \\
\hline $\mathrm{H}(14 \mathrm{~B})$ & 306 & 72 & 1464 & 41 \\
\hline $\mathrm{H}(14 \mathrm{C})$ & 1430 & -251 & 784 & 41 \\
\hline $\mathrm{H}(13 \mathrm{~A})$ & 1881 & -866 & 2788 & 30 \\
\hline $\mathrm{H}(13 \mathrm{~B})$ & 3237 & -536 & 2428 & 30 \\
\hline $\mathrm{H}(7 \mathrm{~A})$ & 1041 & 4271 & 4258 & 48 \\
\hline $\mathrm{H}(7 \mathrm{~B})$ & 1833 & 3106 & 4341 & 48 \\
\hline $\mathrm{H}(7 \mathrm{C})$ & 432 & 3083 & 4583 & 48 \\
\hline $\mathrm{H}(10 \mathrm{~A})$ & 1892 & 3808 & 230 & 36 \\
\hline $\mathrm{H}(10 \mathrm{~B})$ & 3055 & 3795 & 674 & 36 \\
\hline $\mathrm{H}(10 \mathrm{C})$ & 2251 & 4947 & 412 & 36 \\
\hline $\mathrm{H}(15 \mathrm{~A})$ & 3527 & 635 & 503 & 41 \\
\hline $\mathrm{H}(15 \mathrm{~B})$ & 3857 & 1471 & 1060 & 41 \\
\hline $\mathrm{H}(15 \mathrm{C})$ & 3045 & 1939 & 264 & 41 \\
\hline $\mathrm{H}(6 \mathrm{~A})$ & 3798 & 3565 & 2046 & 39 \\
\hline $\mathrm{H}(6 \mathrm{~B})$ & 3423 & 3459 & 3132 & 39 \\
\hline$H(6 C)$ & 3344 & 4674 & 2401 & 39 \\
\hline $\mathrm{H}(11 \mathrm{~A})$ & 3705 & 1415 & 3897 & 46 \\
\hline $\mathrm{H}(11 \mathrm{~B})$ & 4164 & 1259 & 2877 & 46 \\
\hline $\mathrm{H}(11 \mathrm{C})$ & 4147 & 182 & 3758 & 46 \\
\hline $\mathrm{H}(9 \mathrm{~A})$ & -746 & 4722 & 906 & 44 \\
\hline $\mathrm{H}(9 \mathrm{~B})$ & -1274 & 3524 & 1449 & 44 \\
\hline $\mathrm{H}(9 \mathrm{C})$ & -194 & 3589 & 622 & 44 \\
\hline
\end{tabular}


Table 6. Torsion angles [deg] for CpsIrdmpmcl.

\begin{tabular}{|c|c|}
\hline $\mathrm{C}(1)-\operatorname{Ir}(1)-\mathrm{P}(1)-\mathrm{C}(12)$ & $111.3(4)$ \\
\hline$C(5)-\operatorname{Ir}(1)-P(1)-C(12)$ & $139.7(4)$ \\
\hline $\mathrm{C}(4)-\operatorname{Ir}(1)-\mathrm{P}(1)-\mathrm{C}(12)$ & $89.2(10)$ \\
\hline$C(2)-\operatorname{Ir}(1)-P(1)-C(12)$ & $70.6(4)$ \\
\hline $\mathrm{C}(3)-\operatorname{Ir}(1)-\mathrm{P}(1)-\mathrm{C}(12)$ & $43.2(4)$ \\
\hline$P(2)-\operatorname{Ir}(1)-P(1)-C(12)$ & $-129.1(3)$ \\
\hline $\mathrm{Cl}(1)-\operatorname{Ir}(1)-\mathrm{P}(1)-\mathrm{C}(12)$ & $-43.4(3)$ \\
\hline$C(1)-\operatorname{Ir}(1)-P(1)-C(11)$ & $-19.8(4)$ \\
\hline$C(5)-\operatorname{Ir}(1)-P(1)-C(11)$ & $8.7(4)$ \\
\hline$C(4)-\operatorname{Ir}(1)-P(1)-C(11)$ & $-41.8(10)$ \\
\hline$C(2)-\operatorname{Ir}(1)-P(1)-C(11)$ & $-60.5(4)$ \\
\hline$C(3)-\operatorname{Ir}(1)-P(1)-C(11)$ & $-87.8(4)$ \\
\hline$P(2)-\operatorname{Ir}(1)-P(1)-C(11)$ & $99.9(3)$ \\
\hline $\mathrm{Cl}(1)-\operatorname{Ir}(1)-\mathrm{P}(1)-\mathrm{C}(11)$ & $-174.4(3)$ \\
\hline$C(1)-\operatorname{Ir}(1)-P(1)-C(13)$ & $-131.8(3)$ \\
\hline$C(5)-\operatorname{Ir}(1)-P(1)-C(13)$ & $-103.3(3)$ \\
\hline$C(4)-\operatorname{Ir}(1)-P(1)-C(13)$ & $-153.8(10)$ \\
\hline$C(2)-\operatorname{Ir}(1)-P(1)-C(13)$ & $-172.5(3)$ \\
\hline$C(3)-\operatorname{Ir}(1)-P(1)-C(13)$ & $160.1(3)$ \\
\hline$P(2)-\operatorname{Ir}(1)-P(1)-C(13)$ & $-12.1(2)$ \\
\hline $\mathrm{Cl}(1)-\operatorname{Ir}(1)-\mathrm{P}(1)-\mathrm{C}(13)$ & $73.6(2)$ \\
\hline $\mathrm{C}(1)-\operatorname{Ir}(1)-\mathrm{P}(1)-\mathrm{P}(2)$ & $-119.69(18)$ \\
\hline$C(5)-\operatorname{Ir}(1)-P(1)-P(2)$ & $-91 \cdot 2(3)$ \\
\hline $\mathrm{C}(4)-\operatorname{Ir}(1)-\mathrm{P}(1)-\mathrm{P}(2)$ & $-141.7(10)$ \\
\hline $\mathrm{C}(2)-\operatorname{Ir}(1)-\mathrm{P}(1)-\mathrm{P}(2)$ & $-160.38(19)$ \\
\hline $\mathrm{C}(3)-\operatorname{Ir}(1)-\mathrm{P}(1)-\mathrm{P}(2)$ & $172.3(3)$ \\
\hline $\mathrm{Cl}(1)-\operatorname{Ir}(1)-\mathrm{P}(1)-\mathrm{P}(2)$ & $85.70(7)$ \\
\hline $\mathrm{C}(1)-\operatorname{Ir}(1)-\mathrm{P}(2)-\mathrm{C}(14)$ & $-133.7(4)$ \\
\hline$C(5)-\operatorname{Ir}(1)-P(2)-C(14)$ & $-97.5(3)$ \\
\hline$C(4)-\operatorname{Ir}(1)-P(2)-C(14)$ & $-58.8(4)$ \\
\hline $\mathrm{C}(2)-\operatorname{Ir}(1)-\mathrm{P}(2)-\mathrm{C}(14)$ & $-142.5(6)$ \\
\hline$C(3)-\operatorname{Ir}(1)-P(2)-C(14)$ & $-40.3(5)$ \\
\hline$P(1)-\operatorname{Ir}(1)-P(2)-C(14)$ & $128.4(3)$ \\
\hline $\mathrm{Cl}(1)-\operatorname{Ir}(1)-\mathrm{P}(2)-\mathrm{C}(14)$ & $42.1(3)$ \\
\hline$C(1)-\operatorname{Ir}(1)-P(2)-C(15)$ & $-2.5(4)$ \\
\hline$C(5)-\operatorname{Ir}(1)-P(2)-C(15)$ & $33.7(3)$ \\
\hline$C(4)-\operatorname{Ir}(1)-P(2)-C(15)$ & $72.4(4)$ \\
\hline$C(2)-\operatorname{Ir}(1)-P(2)-C(15)$ & $-11 \cdot 3(6)$ \\
\hline$C(3)-\operatorname{Ir}(1)-P(2)-C(15)$ & $90.9(5)$ \\
\hline$P(1)-\operatorname{Ir}(1)-P(2)-C(15)$ & $-100.4(3)$ \\
\hline $\mathrm{Cl}(1)-\operatorname{Ir}(1)-\mathrm{P}(2)-\mathrm{C}(15)$ & $173.3(3)$ \\
\hline $\mathrm{C}(1)-\operatorname{Ir}(1)-\mathrm{P}(2)-\mathrm{C}(13)$ & $109.9(3)$ \\
\hline$C(5)-\operatorname{Ir}(1)-P(2)-C(13)$ & $146.1(3)$ \\
\hline$C(4)-\operatorname{Ir}(1)-P(2)-C(13)$ & $-175.2(3)$ \\
\hline $\mathrm{C}(2)-\operatorname{Ir}(1)-\mathrm{P}(2)-\mathrm{C}(13)$ & $101.1(6)$ \\
\hline$C(3)-\operatorname{Ir}(1)-P(2)-C(13)$ & $-156.7(4)$ \\
\hline$P(1)-\operatorname{Ir}(1)-P(2)-C(13)$ & $12.0(2)$ \\
\hline $\mathrm{Cl}(1)-\operatorname{Ir}(1)-\mathrm{P}(2)-\mathrm{C}(13)$ & $-74.3(2)$ \\
\hline $\mathrm{C}(1)-\operatorname{Ir}(1)-\mathrm{P}(2)-\mathrm{P}(1)$ & $97.9(2)$ \\
\hline$C(5)-\operatorname{Ir}(1)-P(2)-P(1)$ & $134.09(19)$ \\
\hline $\mathrm{C}(4)-\operatorname{Ir}(1)-\mathrm{P}(2)-\mathrm{P}(1)$ & $172.8(2)$ \\
\hline $\mathrm{C}(2)-\operatorname{Ir}(1)-\mathrm{P}(2)-\mathrm{P}(1)$ & $89.1(5)$ \\
\hline $\mathrm{C}(3)-\operatorname{Ir}(1)-\mathrm{P}(2)-\mathrm{P}(1)$ & $-168.8(4)$ \\
\hline
\end{tabular}




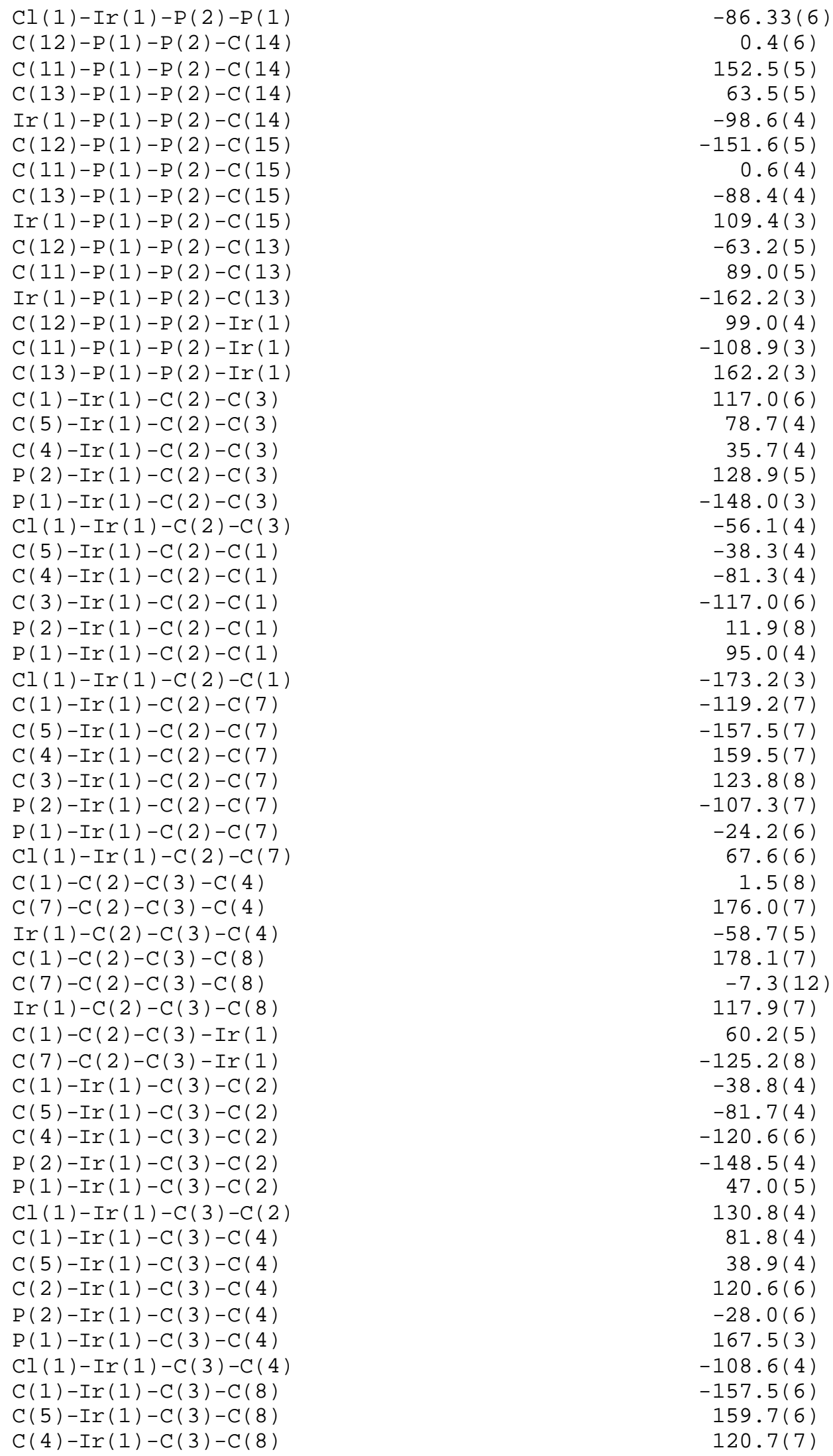




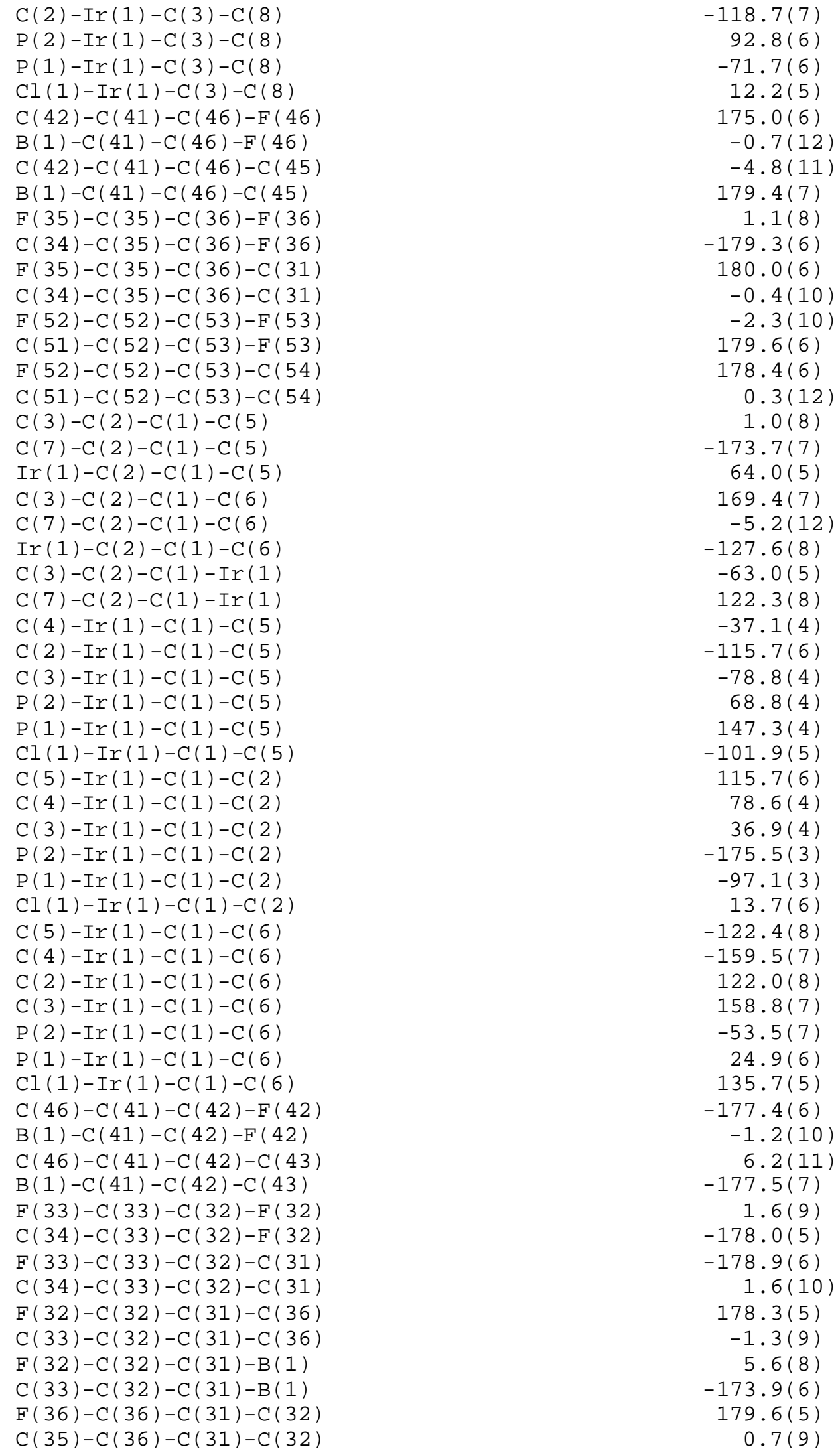




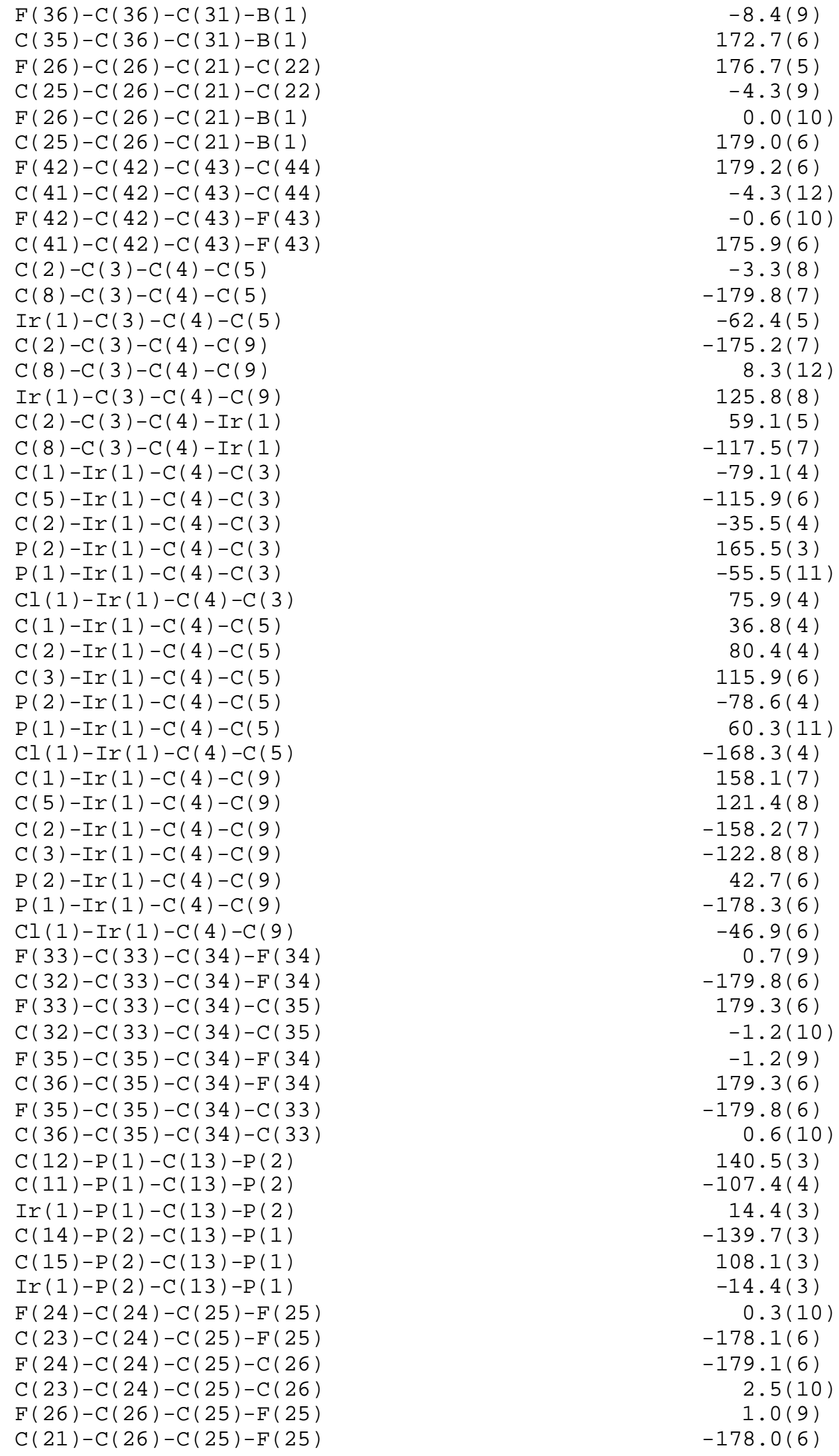

$-8.4(9)$

$172.7(6)$

$176.7(5)$

$-4.3(9)$

$0.0(10)$

$179.0(6)$

$179.2(6)$

$-4.3(12)$

$-0.6(10)$

$175.9(6)$

$-3.3(8)$

$-179.8(7)$

$-62.4(5)$

$-175.2(7)$

$8.3(12)$

$125.8(8)$

$59.1(5)$

$-117.5(7)$

$-79.1(4)$

$-115.9(6)$

$-35.5(4)$

$165.5(3)$

$-55.5(11)$

$75.9(4)$

$36.8(4)$

$80.4(4)$

$115.9(6)$

$-78.6(4)$

$60.3(11)$

$-168.3(4)$

$158.1(7)$

$121.4(8)$

$-158.2(7)$

$-122.8(8)$

$42.7(6)$

$-178.3(6)$

$-46.9(6)$

$0.7(9)$

$-179.8(6)$

$179.3(6)$

$-1.2(10)$

$-1.2(9)$

$179.3(6)$

$-179.8(6)$

$0.6(10)$

$140.5(3)$

$-107.4(4)$

$14.4(3)$

-139.7 (3)

108.1 (3)

$-14.4(3)$

$0.3(10)$

-178.1 (6)

-179.1 (6)

$2.5(10)$

$1.0(9)$

$-178.0(6)$ 


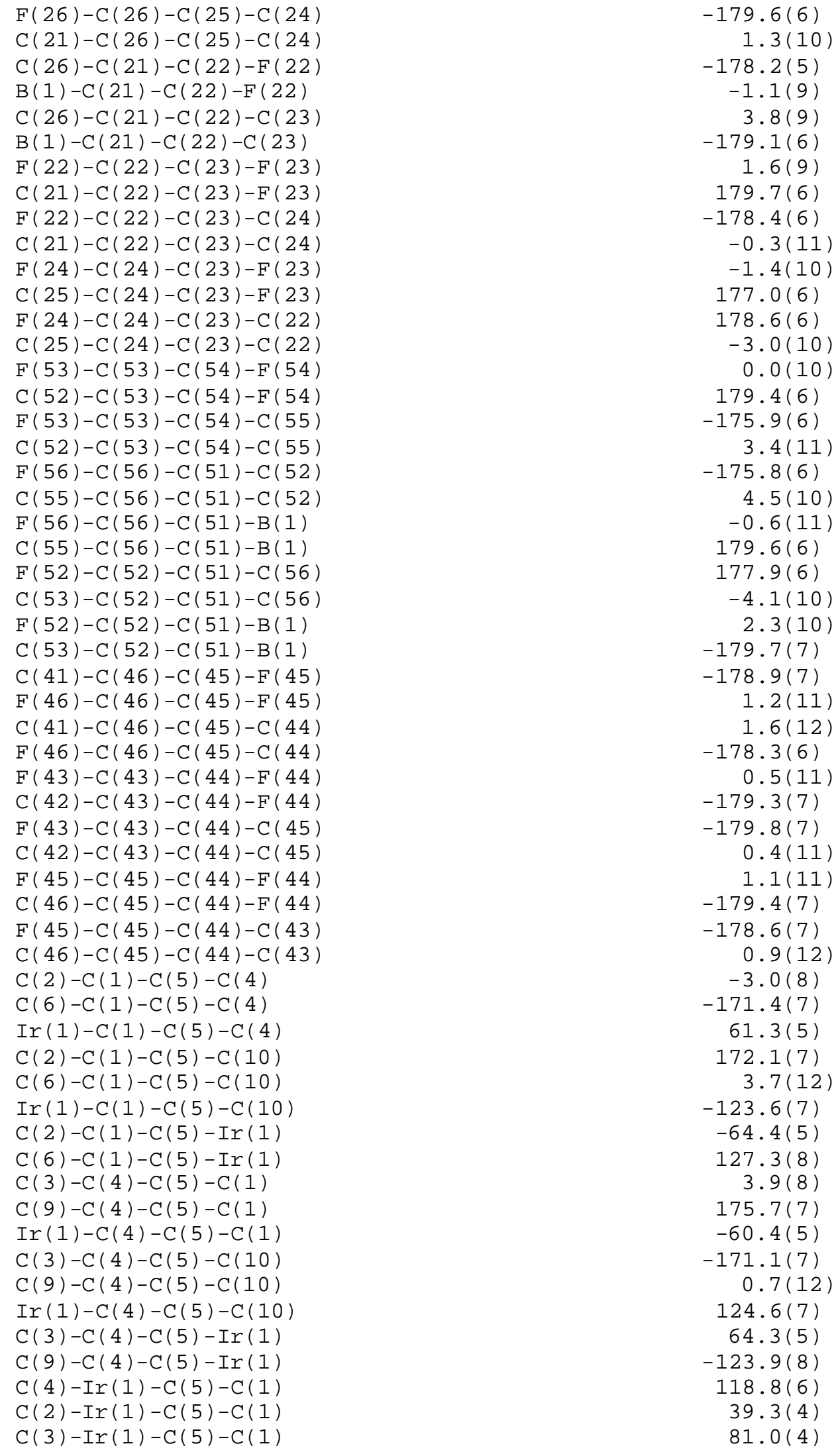




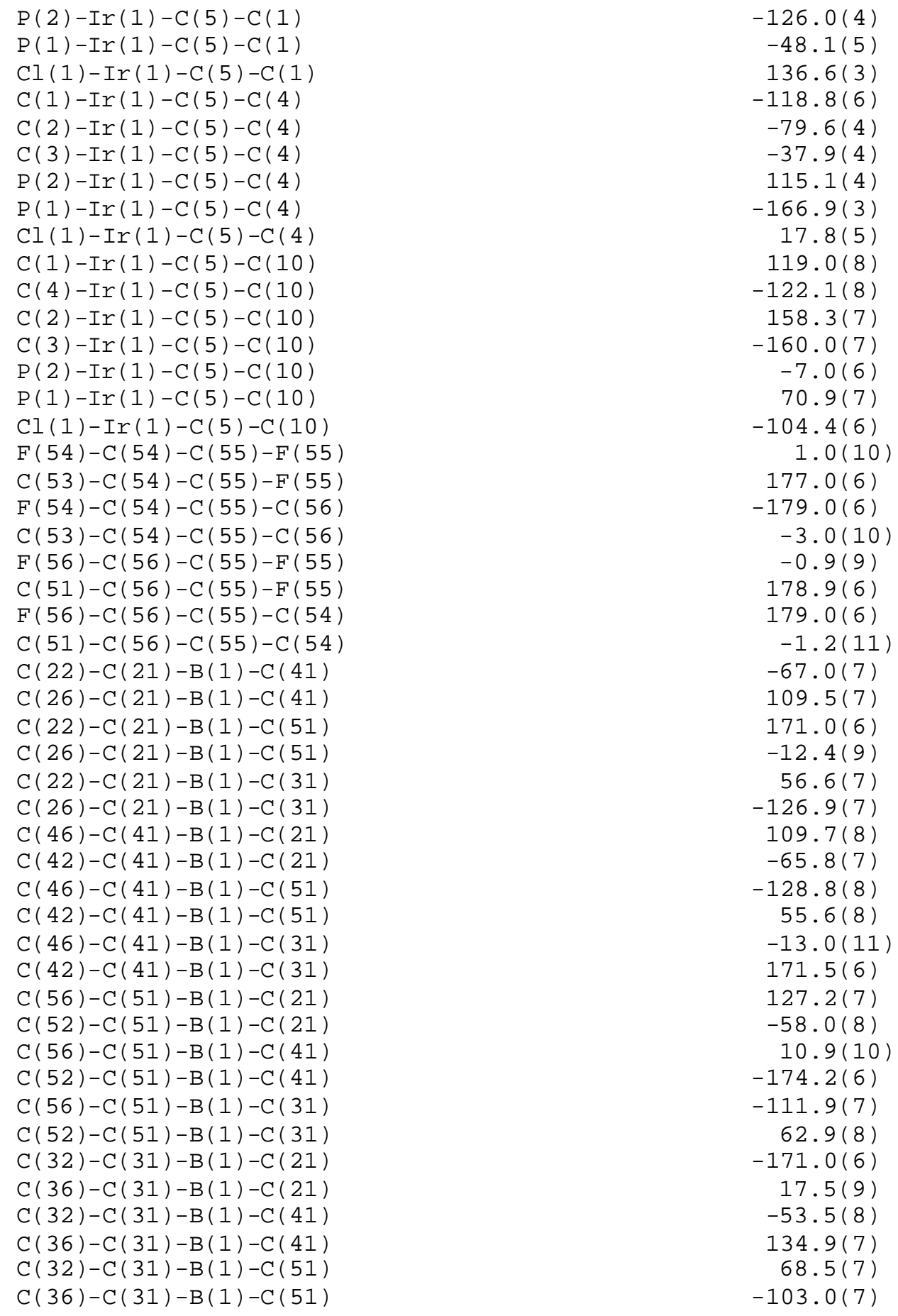

Symmetry transformations used to generate equivalent atoms: 
References:

\author{
Instrument: Nonius KappaCCD \\ Nonius (1997). KappaCCD Operations \\ Manual, Delft. \\ Data Collection Software: Collect, (1998), Data Collection Software, \\ Nonius.
}

Data Reduction: HKL2000

Otinowski, Z., Minor, W., (1996). Processing of X-ray Diffraction Data Collected in Oscillation Mode, Methods in Enzymology, 276, 307-326., C. W. Carter, Jr., R. M. Sweet, Eds., Academic Press.

Cell Refinement: HKL Scalepack (Otwinowski \& Minor 1997)

ibid.

Structure Solution: SIR97

Altomare, A., Cascarano, G., Giacovazzo, C., Burla, M.C., Polidori, G., Camalli, M., (1994)., SIR. J. Appl. Cryst. 27, 435-442 .

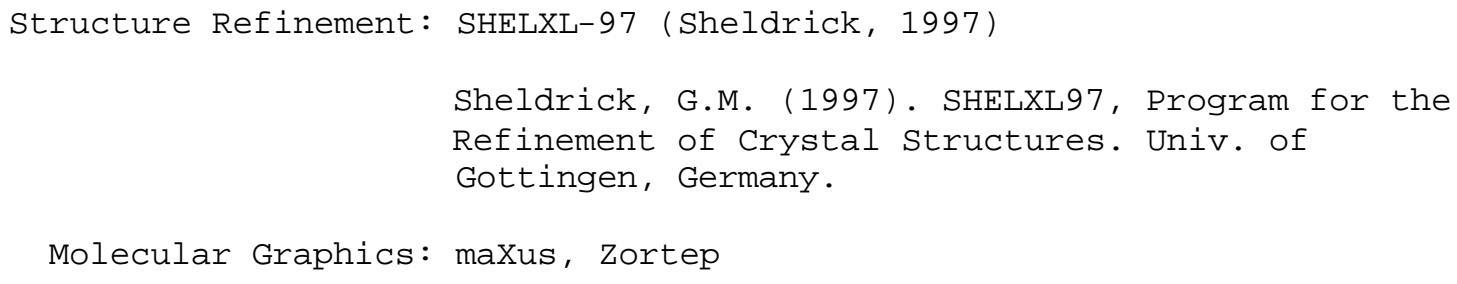

